\title{
S-nitrosylation of transglutaminase 2 impairs fatty acid-stimulated contraction in hypertensive cardiomyocytes
}

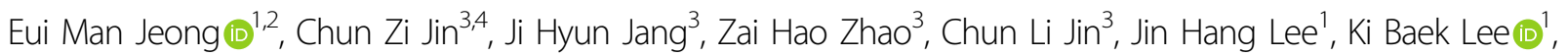
Sung Joon Kim ${ }^{3}$ In-Gyu Kim ${ }^{1,2}$ and Yin Hua Zhang ${ }^{3,4,5}$

\begin{abstract}
The myocardium in hypertensive heart exhibits decreased fatty acid utilization and contractile dysfunction, leading to cardiac failure. However, the causal relationship between metabolic remodeling and cardiomyocyte contractility remains unestablished. Transglutaminase 2 (TG2) has been known to promote ATP production through the regulation of mitochondrial function. In this study, we investigated the involvement of TG2 in cardiomyocyte contraction under fatty acid supplementation. Using TG2 inhibitor and TG2-deficient mice, we demonstrated that fatty acid supplementation activated TG2 and increased ATP level and contractility of cardiac myocyte from the normal heart. By contrast, in cardiac myocytes from angiotensin-II-treated rats and mice, the effects of fatty acid supplementation on TG2 activity, ATP level, and myocyte contraction were abolished. We found that TG2 was inhibited by S-nitrosylation and its level increased in hypertensive myocytes. Treatment with inhibitor for neuronal NOS restored fatty acidinduced increase of TG2 activity and myocyte contraction. Moreover, intracellular $\mathrm{Ca}^{2+}$ levels were increased by fatty acid supplementation in both normal and hypertensive myocytes, showing that S-nitrosylation of TG2 but not alteration of intracellular $\mathrm{Ca}^{2+}$ levels is responsible for contractile dysfunction. These results indicate that TG2 plays a critical role in the regulation of myocyte contractility by promoting fatty acid metabolism and provide a novel target for preventing contractile dysfunction in heart with high workload.
\end{abstract}

\section{Introduction}

Myocardial ATP production in the heart is predominantly derived from fatty acid oxidation, which accounts for up to $90 \%$ of the ATP required for continual contraction $^{1}$. However, in response to pressure overload, myocytes exhibit a decreased dependence on fatty acid oxidation for ATP production and a concomitant increase in glucose uptake and glycolysis ${ }^{2,3}$. This switch in substrate from fatty acids to glucose has been suggested to play a crucial role in the development of pressure

\footnotetext{
Correspondence: I.-G. Kim(igkim@snu.ac.kr) or Y. H. Zhang(yinzhang@snu.ac.kr) ${ }^{1}$ Department of Biochemistry and Molecular Biology, Seoul National University College of Medicine, Jongno-gu, Seoul, Republic of Korea

${ }^{2}$ Institute of Human-Environment Interface Biology, Seoul National University College of Medicine, Jongno-gu, Seoul, Republic of Korea

Full list of author information is available at the end of the article

These authors contributed equally: Eui Man Jeong, Chun Zi Jin.
}

overload-induced cardiac hypertrophy, fibrosis, and contractile dysfunction ${ }^{2}$; however, the mechanisms underlying the decrease in fatty acid utilization observed in the pressure-overloaded heart have not been fully understood.

Transglutaminase 2 (TG2) is a calcium-dependent enzyme that catalyzes the formation of covalent bonds between peptide-bound glutamine and various primary amines, such as $\gamma$-amino group of peptide-bound lysine or polyamines, thereby producing cross-linked or polyaminated proteins, respectively ${ }^{4}$. These TG2-mediated modifications modulate the activities of a number of substrate proteins ${ }^{5}$. Elevated levels of TG2 activity have been shown to be associated with increased cell survival, inflammatory response, and extracellular matrix formation $^{6}$. In the cardiovascular system, TG2 has been reported to be involved in the development of cardiac 
hypertrophy, vessel remodeling, and age-related vascular stiffness under various conditions ${ }^{7-10}$. Interestingly, TG2 is implicated in the regulation of ATP synthesis and transport via modification of respiratory complexes and the ADP/ATP transporter, respectively, in the mitochondria of skeletal muscle and myocardium, thereby increasing exercise tolerance ${ }^{11,12}$. However, the role of TG2 in the heart during conditions of pressure overload, especially in relation to ATP production from fatty acid oxidation in the mitochondria, has not been investigated.

In the myocardium, three isoforms of nitric oxide synthase (NOS), neuronal NOS (nNOS), endothelial NOS (eNOS), and inducible NOS, which are expressed in different subcellular organelles, play a role in the regulation of cardiac function via the production of nitric oxide ${ }^{13}$. Recently, we have shown that nNOS expression is significantly upregulated in the left ventricular (LV) myocytes of Ang II-induced hypertensive rats, whereas the protein level of eNOS is downregulated ${ }^{14}$. Similar nNOS upregulation has been reported in hypertrophic or failing myocardium in which fatty acid-dependent ATP production is impaired ${ }^{15}$.

Under nitrosative stress conditions, TG2 is known to be posttranslationally modified by $S$-nitrosylation of cysteine residues, resulting in the inhibition of its activity ${ }^{8,16}$. Considering the role of TG2 in promoting mitochondrial ATP production, these findings suggest the involvement of $\mathrm{nNOS}$ in the metabolic regulation of pressureoverloaded myocardium. Therefore, in the present study, we sought to investigate the role of TG2 and nNOS in fatty acid-induced ATP production and myocyte contraction in the normal and hypertensive heart.

\section{Materials and methods \\ Animals}

Sprague-Dawley rats (12 weeks, male, Orient Bio Inc, Seongnam-Si, Republic of Korea) and TG2-null mice ${ }^{17}$ $\left(\mathrm{TG}^{-1-}, 12\right.$ weeks, male, backcrossed to $\mathrm{C} 57 \mathrm{BL} / 6 \mathrm{~J}$ mice for 12 generations) were used. Briefly, rats or mice were anesthetized with isoflurane. An osmotic minipump (Alzet model 2004, DURECT Corporation, Cupertino, CA) containing Ang II (infusion rate $125 \mathrm{ng} / \mathrm{min} / \mathrm{kg}$ ) was implanted in the midscapular region under sterile conditions for 4 weeks. Rats and mice were paired with a sham-operated group. Our previous results showed that Ang II infusion significantly increased both systolic and diastolic blood pressure at 4 weeks ${ }^{13}$.

The study protocol was in accordance with the Guide for the Care and Use of Laboratory Animals published by the US National Institutes of Health (NIH Publication No. 85-23, revised 1996) and also conformed to the guidelines of the Institutional Animal Care and Use Committee (IACUC), Seoul National University (IACUC approval No.: SNU-101012-3).

\section{Isolation of LV myocytes from the rat and mouse hearts}

LV myocytes were isolated using a standard enzymatic dispersion technique ${ }^{14}$. Briefly, the rats were anesthetized with pentobarbital sodium $(30 \mathrm{mg} / \mathrm{kg}$, intraperitoneal) or mice were killed humanely with cervical dislocation. Hearts were quickly extracted and mounted onto the Langendorff perfusion system to perfuse with a nominal $\mathrm{Ca}^{2+}$-free solution comprising $135 \mathrm{mM} \mathrm{NaCl}, 5.4 \mathrm{mM}$ $\mathrm{KCl}, 3.5 \mathrm{mM} \mathrm{MgCl}_{2}, 5 \mathrm{mM}$ glucose, $5 \mathrm{mM}$ Hepes, $0.4 \mathrm{mM}$ $\mathrm{Na}_{2} \mathrm{HPO}_{4}$, and $20 \mathrm{mM}$ taurine, $\mathrm{pH}$ 7.4, followed by further perfusion for $8 \mathrm{~min}$ with the same solution with enzymes $(1 \mathrm{mg} / \mathrm{ml}$ collagenase, Worthington Biochemical Co., Lakewood, NJ; $0.133 \mathrm{mg} / \mathrm{ml}$ protease, Sigma Aldrich, Saint Louis, MO; $1.65 \mathrm{mg} / \mathrm{ml}$ bovine serum albumin; 0.05 $\left.\mathrm{mM} \mathrm{Ca}^{2+}\right) . \mathrm{LV}$ was dissected in a collagenase-containing flask for further 5- and 10-min digestion periods. Isolated myocytes were washed and resuspended in storage solution $\left(120 \mathrm{mM} \mathrm{NaCl}, 5.4 \mathrm{mM} \mathrm{KCl}, 5 \mathrm{mM} \mathrm{MgSO}_{4}, 0.2 \mathrm{mM}\right.$ $\mathrm{CaCl}_{2}, 5 \mathrm{mM}$ Na-pyruvate, $5.5 \mathrm{mM}$ glucose, $20 \mathrm{mM}$ taurine, $10 \mathrm{mM}$ HEPES, and $29 \mathrm{mM}$ mannitol, $\mathrm{pH} 7.4$ ). The myocyte suspension was stored at room temperature and cells were used within $8 \mathrm{~h}$ of isolation.

\section{Measurement of LV myocyte contraction}

LV myocytes were superfused with normal tyrode solution containing $141.4 \mathrm{mM} \mathrm{NaCl}, 4 \mathrm{mM} \mathrm{KCl}, 0.33 \mathrm{mM}$ $\mathrm{NaH}_{2} \mathrm{PO}_{4}, 1 \mathrm{mM} \mathrm{MgCl}, 10 \mathrm{mM}$ HEPES, $5.5 \mathrm{mM}$ Glucose, $1.8 \mathrm{mM} \mathrm{CaCl}_{2}$, and $14.5 \mathrm{mM}$ manitol, $\mathrm{pH} 7.4$. Changes in sarcomere length were measured in $\mathrm{LV}$ myocytes (field stimulated at $2 \mathrm{~Hz}$ ) using a videosarcomere detection system (IonOptix Corp, Westwood, $\mathrm{MA})$. The difference between diastolic and systolic sarcomere length ( $\Delta$ sarcomere shortening) was compared between LV myocytes from the control and Ang II-treated groups.

\section{TG activity assay}

For in vitro TG activity assay in lysates of isolated LV myocytes, microtiter plate method was utilized using $N$, $N^{3}$-dimethylcasein and biotinylated pentylamine (BP, Pierce, Rockford, IL) as substrates ${ }^{18}$. Briefly, $10 \mu \mathrm{g}$ of $N$, $N^{\prime}$-dimethylcasein in $100 \mu \mathrm{l}$ coating buffer $(50 \mathrm{mM}$ Tris-Cl, pH 7.5, $150 \mathrm{mM} \mathrm{NaCl}, 5 \mathrm{mM}$ EGTA, $5 \mathrm{mM}$ EDTA) was added to each well of a 96-well microtiter plate (Nunc, Roskilde, Denmark) and incubated at $4{ }^{\circ} \mathrm{C}$ overnight. After washing with phosphate-buffered saline with Tween 20 , cell lysate (15 $\mu \mathrm{g}$ of proteins) and $50 \mu \mathrm{M}$ BP in $50 \mu \mathrm{l} \mathrm{TG}$ reaction buffer $(50 \mathrm{mM}$ Tris-Cl, pH 7.5, $2 \mathrm{mM} \mathrm{CaCl}_{2}, 5 \mathrm{mM}$ dithiothreitol, and $1 \%$ Triton X100) were added to the wells and incubated for $1 \mathrm{~h}$ at $37^{\circ} \mathrm{C}$. After incubating with streptavidin-horseradish peroxidase (SA-HRP) for $1 \mathrm{~h}$ at room temperature, TG activity was quantified by reaction with ophenylenediamine dihydrochloride (OPD) and 
measurement of absorbance at $492 \mathrm{~nm}$ on microplate spectrophotometer (Molecular Devices, Sunnyvale, CA) after stopping the reaction by adding $1 \mathrm{M} \mathrm{H}_{2} \mathrm{SO}_{4}$.

Intracellular TG activity assay was performed by the microtiter plate method ${ }^{18}$. In brief, LV myocytes were incubated with storage solution containing $1 \mathrm{mM}$ BP for $1 \mathrm{~h}$ at $37^{\circ} \mathrm{C}, 5 \% \mathrm{CO}_{2}$. Cells were harvested and disrupted in lysis buffer (50 mM Tris, $\mathrm{pH} 7.5,150 \mathrm{mM} \mathrm{NaCl}, 1 \mathrm{mM}$ EDTA, 1\% Triton X-100, and protease inhibitor cocktail, Roche Korea Co., Seoul, Republic of Korea). The homogenate was centrifuged at $12,000 \times g, 4{ }^{\circ} \mathrm{C}$, for $10 \mathrm{~min}$. The protein concentration of the supernatant was determined by BCA method. The cell lysates $(5-10 \mu \mathrm{g} /$ well $)$ were added to each well of a 96-well microtiter plate and coated. After incubation of SA-HRP, intracellular TG activity was quantified by reaction with OPD. The lysates of cells, which were cultured with same conditions without BP treatment, were used as the blank samples.

\section{Measurement of myocyte ATP level}

Intracellular ATP level of LV myocytes was measured using Luminescence Assay system (ATPlite ${ }^{\mathrm{TM}}$, Perkin Elmer, Waltham, MA). Prepared cells were snap-frozen in liquid nitrogen, followed by lysis with $1 \times$ mammalian cell lysis solution. Protein concentration was determined using Bradford protocol. Then $150 \mu \mathrm{l}$ of cell lysate and 50 $\mu \mathrm{l}$ substrate solution were added to the wells, and the microplate was shaken for $5 \mathrm{~min}$ in an orbital shaker at $700 \mathrm{rpm}$. The plate was placed in the dark for $10 \mathrm{~min}$ prior to luminescence measurement. Luminescence of the mixture was measured using a microplate plate reader (Tecan Microplate Reader: Multi-Mode Infinite M200, San Jose, CA).

JC-1 assay for the mitochondrial transmembrane potential

The change of mitochondrial membrane potential (MMP) in isolated myocytes was measured using JC-1 dye (eBioscience $^{\mathrm{TM}}$, Carlsbad, CA) as described below. Isolated myocytes were resuspended in normal tyrode solution and treated with 1 or $2 \mu \mathrm{M}$ Ang II in the presence of $0.5 \mathrm{mM}$ cystamine for $3 \mathrm{~h}$ or $100 \mu \mathrm{M}$ palmitic acid (PA) for $30 \mathrm{~min}$ at $37^{\circ} \mathrm{C}, 5 \% \mathrm{CO}_{2}$ in 96-well black plate ( $\mu$ CLEAR, Greiner bio-one, Frickenhausen, Germany). Cells were loaded with $10 \mu \mathrm{g} / \mathrm{ml}$ of JC- 1 for $10 \mathrm{~min}$ at $37^{\circ}$ $\mathrm{C}, 5 \% \mathrm{CO}_{2}$, shaking every $2 \mathrm{~min}$. Then the solution was removed and fresh normal tyrode solution was added. Cells were observed using Operetta High-Content Imaging System (Perkin Elmer, Waltham, MA). JC-1 aggregates were detected at $560-580 \mathrm{~nm}$ excitation and 590-640 nm emission, and monomers were detected at 460-490 $\mathrm{nm}$ excitation and 500-550 nm emission. JC-1 ratio was calculated using the ImageJ software (National Institutes of Health, Bethesda, MD; https://imagej.nih. gov/ij/).

\section{S-nitrosylation of TG2}

The amount of $S$-nitrosylated TG2 in myocytes was determined by biotin-switch method. The $S$-nitrosylated cysteine residues in TG2 were biotinylated using an $S$ nitrosylated Protein Detection Assay Kit (Cayman Chemical Co., Ann Arbor, MI) according to the manufacturer's instructions. Biotinylated proteins were isolated using streptavidin-conjugated magnetic beads (Dynal Biotech, Oslo, Norway). Then beads were washed with a buffer containing $50 \mathrm{mM}$ Tris-Cl, $\mathrm{pH} 8.0 ; 150 \mathrm{mM} \mathrm{NaCl}$, $1 \mathrm{mM}$ EDTA, and $1 \%$ Triton X-100. TG2 was detected using anti-TG2 polyclonal antibodies (Neomarkers, Fremont, CA). Total TG2 levels of the samples were determined before isolation of biotinylated proteins. The $S$ nitrosylated vs. total TG2 ratio was determined using the ImageJ software.

\section{Measurement of $\mathrm{Ca}^{2+}$ transients}

The $\mathrm{Ca}^{2+}$ transients were evaluated in Fura-2AMloaded $(5 \mu \mathrm{mol} / \mathrm{l}$, Molecular Probes, Eugene, OR) LV myocytes as the ratio of F360/F380 (field stimulation at 2 $\mathrm{Hz}$, IonOptix Corp, Westwood, MA). The amplitude of $\mathrm{Ca}^{2+}$ transients (the difference between diastolic and peak $\mathrm{Ca}^{2+}$ fluorescence) was calculated after the subtraction from the background. Total $\mathrm{Ca}^{2+}$ level during one stimulation period was shown as an integral of $\mathrm{Ca}^{2+}$ signal. Measurements from at least 10 steady-state contractions and $\mathrm{Ca}^{2+}$ transients were averaged for each myocyte for each stage of the experimental protocols. All experiments were carried out at $36 \pm 1{ }^{\circ} \mathrm{C}$.

\section{Statistics}

Data are expressed as means \pm S.D. and $n$ indicates the number of cells used. For all comparisons, cells were obtained from a minimum of three hearts per treatment group per protocol. Data were analyzed using one-way or two-way analyses of variance or Student's unpaired $t$-test. A value of $P<0.05$ was considered statistically significant.

\section{Results}

TG2 is required for fatty acid-induced increase of cardiomyocyte contractility

First, the expression of TG2 in the murine cardiac myocytes was investigated. Western blot analysis indicated that TG2 protein was expressed in the LV myocytes from rat and wild-type mice but not in the myocytes from $\mathrm{TG}^{-1-}$ mice (Fig. 1a). TG activity was about $10 \%$ in LV myocytes from $\mathrm{TG}^{-1-}$ mice compared to those from wild-type mice (Fig. 1b), indicating that TG2 is the major isoform that contributes to TG activity in murine heart muscle cells. Previous reports have shown that TG2 promotes ATP production in the mitochondria of cardiomyocytes $^{11}$. Therefore, we examined the effect of fatty acid supplementation on the levels of intracellular TG2 

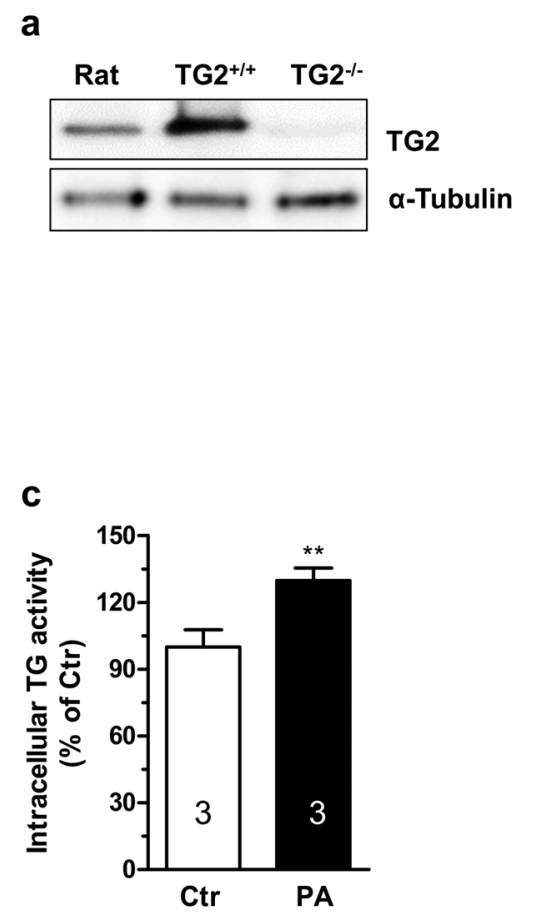

e

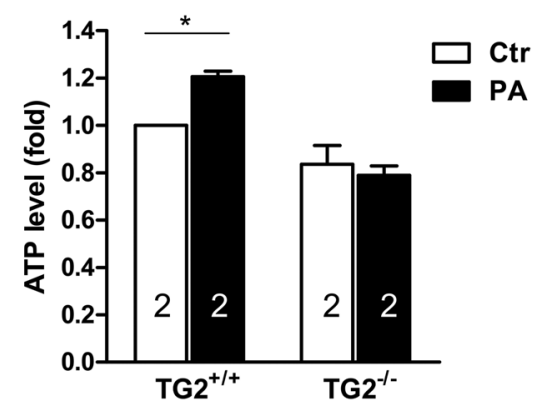

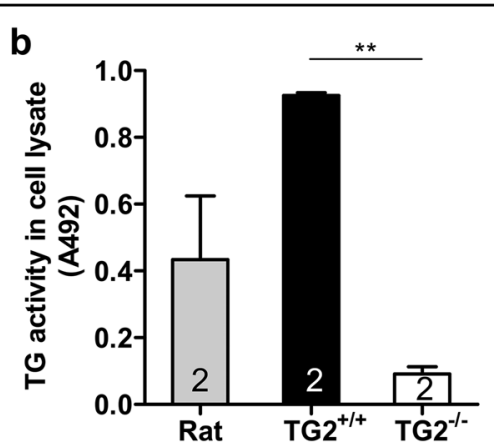

d

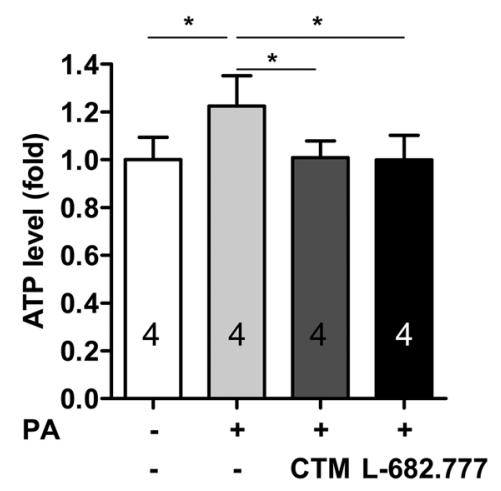

Fig. 1 Palmitic acid supplementation activates TG2 in murine cardiomyocytes. a, b TG2 protein level (a) and TG2 activity (b) were assessed in lysates of left ventricular (LV) myocytes isolated from rat, wild-type, and TG2-deficient mice. c Effects of palmitic acid supplementation (PA, $100 \mu M$ ) on intracellular TG2 activity. d ATP levels in PA-treated LV myocytes from rat hearts in the absence or presence of cystamine (CTM, 0.5 mM) or L-682.777 $(100 \mu \mathrm{M})$, inhibitors of TG2. e ATP levels in PA-treated LV myocytes from wild-type and TG2-deficent mice. Data represent mean \pm S.D. ${ }^{*} P<0.05,{ }^{* *} P<0.01$ compared with control

activity and ATP production in cardiac myocytes. Supplementation of PA $(100 \mu \mathrm{M})$ significantly increased intracellular TG2 activity (Fig. 1c) and ATP level (Fig. 1d) in LV myocytes from the rat hearts. PA-dependent increase of ATP level in cardiac myocytes was decreased by treatment of TG2 inhibitors, cystamine, or L-682.777 (Fig. 1d) and by TG2 ablation (Fig. 1e).

Under the same experimental conditions, PA supplementation increased sarcomere shortening of LV myocytes from the rat hearts $(P<0.001$ between control and PA, $n=26)$. However, inhibition of TG2 activity by treatment with cystamine abolished the effect of PA on myocyte sarcomere shortening ( $P=0.8, n=4$, Fig. 2a). Treatment with L-682.777, a more specific TG2 inhibitor with fewer off-target effects compared to cystamine, also abrogated the effect of PA on myocyte sarcomere shortening $(P=0.01$ in control, $n=4$ vs. $P=0.105$ in L682.777, $n=7$, Fig. 2b). Moreover, PA supplementation increased contraction in LV myocytes from wild-type mice but not in the myocytes from $\mathrm{TG}^{-1-}$ mice $(P=$ 0.007 in TG2 ${ }^{+/+}, n=19$ vs. $P=0.9$ in $\mathrm{TG}^{-1-}, n=7$, Fig. 2c), demonstrating that TG2 mediates PA-induced potentiation of myocyte contraction by increasing intracellular ATP levels. These results indicate that TG2 is 
a
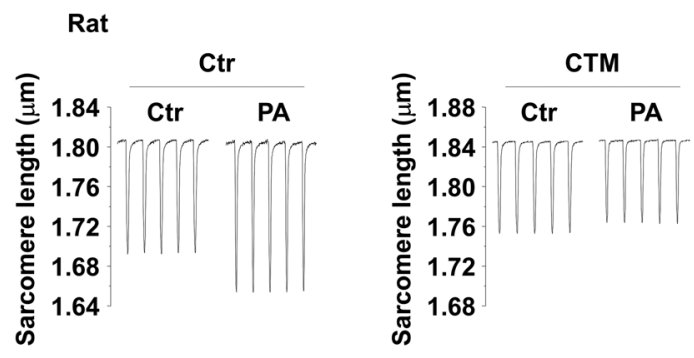

b
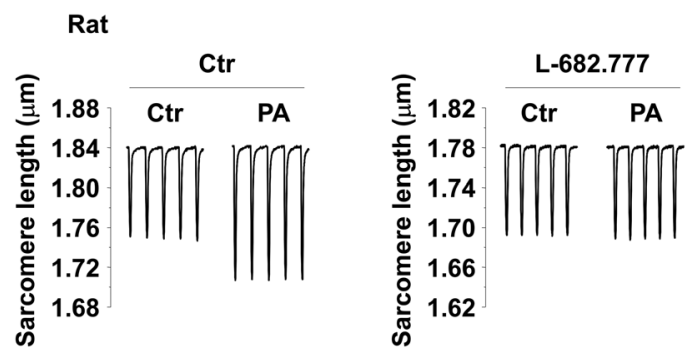

C

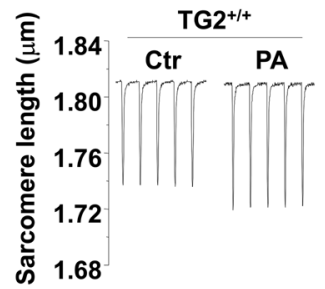

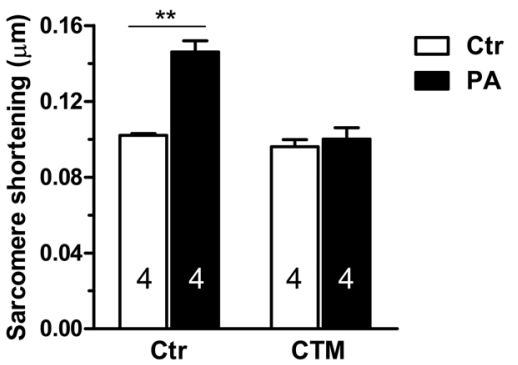
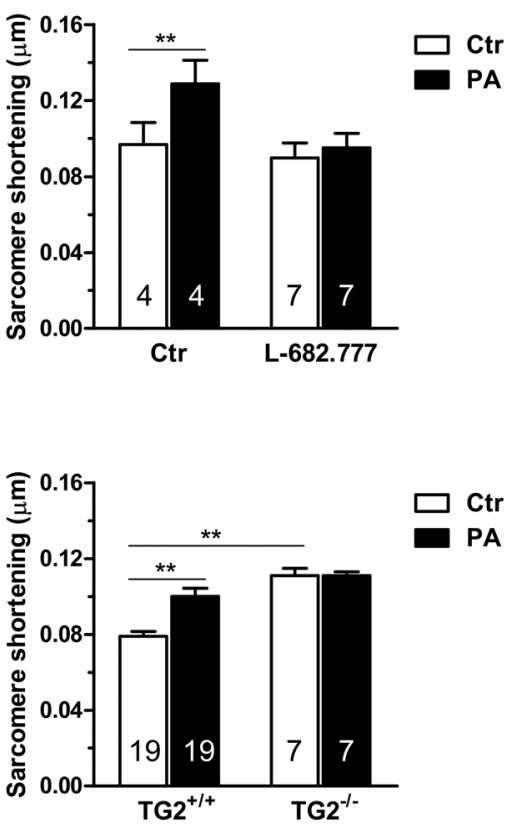

Fig. 2 TG2 mediates palmitic acid-dependent increase of cardiomyocyte contractility. a Sarcomere length of LV myocytes from rats treated with PA was monitored with a video-sarcomere detection system in the absence or presence of CTM (0.5 mM), an inhibitor of TG2 (left). The difference between diastolic and systolic sarcomere length ( $\triangle$ sarcomere shortening) was compared (right). b Effect of PA supplementation on sarcomere length in LV myocytes in the absence or presence of L-682.777 (100 $\mu \mathrm{M})$, an inhibitor of TG2. c Effect of PA supplementation on sarcomere length in LV myocytes from wild-type and TG2-deficient mice. A representative graph is shown. Data represent mean \pm S.D. ${ }^{* *} P<0.01$ compared with control

involved in the regulation of myocyte contraction by promoting fatty acid utilization in the normal heart.

\section{TG2-dependent and fatty acid-induced contraction is} abolished in myocytes in the pressure-overloaded heart

In order to explore the functional role of TG2 in the heart under conditions of increased workload, we induced hypertension in rats and mice by infusion of Ang II for 4 weeks and examined PA-dependent contraction in isolated LV myocytes. Unexpectedly, as shown in Fig. 3, the positive inotropic effect of PA was absent in LV myocytes from Ang II-treated rats $(P=0.5, n=27$, Fig. 3a), Ang II-treated wild type mice $(P=0.8, n=6)$, and Ang II-treated TG $2^{-1-}$ mice $(P=0.8, n=7$, Fig. 3b). In addition, neither intracellular TG2 activity nor ATP levels were increased by PA supplementation in LV myocytes from Ang II-treated rats (TG2 activity: $P=0.67$, $n=4$, Fig. 3c; ATP level: $P=0.5, n=21$, Fig. $3 \mathrm{~d}$ ). These results demonstrate that Ang II treatment abolishes TG2dependent and PA-induced increase in intracellular ATP levels and myocyte contraction.

\section{TG2 is required for fatty acid-dependent polarization of mitochondrial membrane in LV myocytes}

Production of ATP from fatty acid oxidation is related with an increase of MMP. To investigate whether TG2 is 

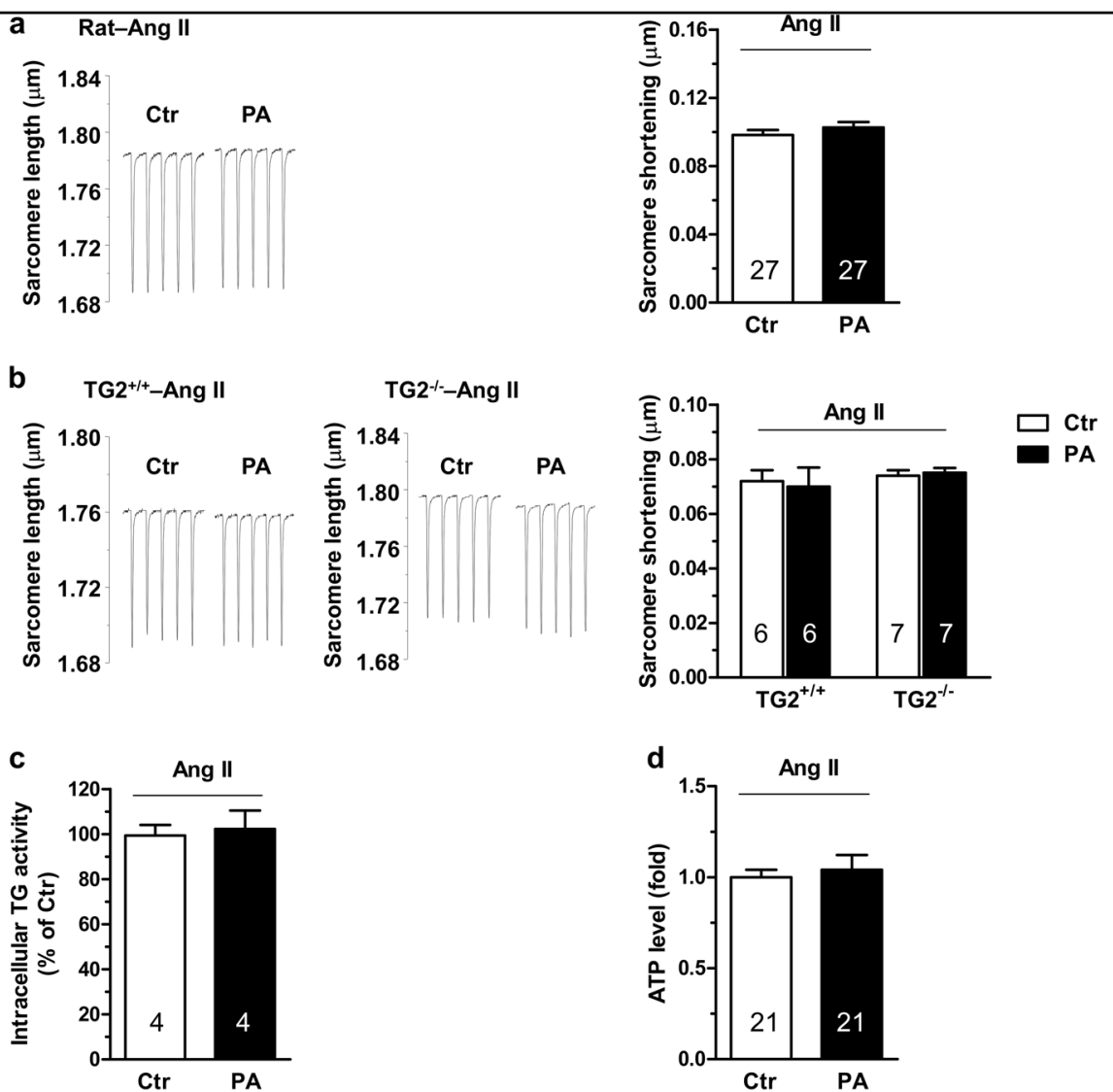

Fig. 3 Palmitic acid-dependent contraction is impaired in cardiomyocytes from Ang II-treated rats and mice. a, b A representative graph showing sarcomere length in LV myocytes from Ang II-treated rats (a) and wild-type and TG2-deficient mice (b). The difference between diastolic and systolic sarcomere length ( $\triangle$ sarcomere shortening) was compared (right). c, d Effects of PA supplementation on intracellular TG2 activity levels (c) and ATP levels (d) in LV myocytes from Ang II-treated rats. Data represent mean \pm S.D.

involved in the regulation of MMP of LV myocytes, we measured the change of MMP in PA-supplemented or Ang II-treated myocytes using JC-1, a green fluorescent dye which exhibits MMP-dependent fluorescence emission shift from green to red by forming J-aggregates. When stained with JC-1, the ratio of red to green fluorescence (JC-1 ratio) of wild-type myocytes was higher than that of TG2-deficient myocytes. Under the same experimental conditions, PA supplementation significantly increased JC-1 ratio of wild-type myocytes but not that of TG2-deficient myocytes (Fig. 4a). By contrast, treatment with Ang II resulted in a concentrationdependent decrease in JC-1 ratio of wild-type myocytes but had no effect on JC-1 ratio of TG2-deficient myocytes (Fig. 4b). Moreover, in LV myocytes from the rat hearts, treatment with Ang II and/or cystamine significantly decreased JC-1 ratio (Fig. 4c). These results indicate that TG2 promotes PA-dependent polarization of mitochondrial membrane in LV myocytes, contributing to ATP production.
TG2 is inhibited by S-nitrosylation in LV myocytes during pressure overload

We have demonstrated that nNOS expression and activity are increased in LV myocytes from Ang II-treated hypertensive rat hearts ${ }^{14}$. Additionally, nitric oxide (NO) is known to inactivate TG2 via $S$-nitrosylation, putatively of the active site cysteine residue ${ }^{16}$. Therefore, we examined $S$-nitrosylation levels of TG2 using a biotinswitch method. Western blot analysis revealed that, although both levels of $S$-nitrosylated TG2 and total TG2 tended to increase in LV myocytes under Ang II-induced hypertensive condition, the ratio of $S$-nitrosylated TG2/total TG2 was significantly higher in LV myocytes from hypertensive rats than that in myocytes from sham controls $(P<0.05)$. Treatment with SMTC $(100 \mathrm{nM}$, $30 \mathrm{~min}$ ), a specific nNOS inhibitor, significantly reduced $S$-nitrosylation levels of TG2 in myocytes from hypertensive rats $(P<0.05$, control vs. Ang II rat treated with SMTC) but did not affect the levels of TG2 $S$-nitrosylation in myocytes from sham controls $(P=0.59$, Fig. 5a). Moreover, SMTC or L-VNIO (100 $\mu \mathrm{M}, 30 \mathrm{~min})$, 


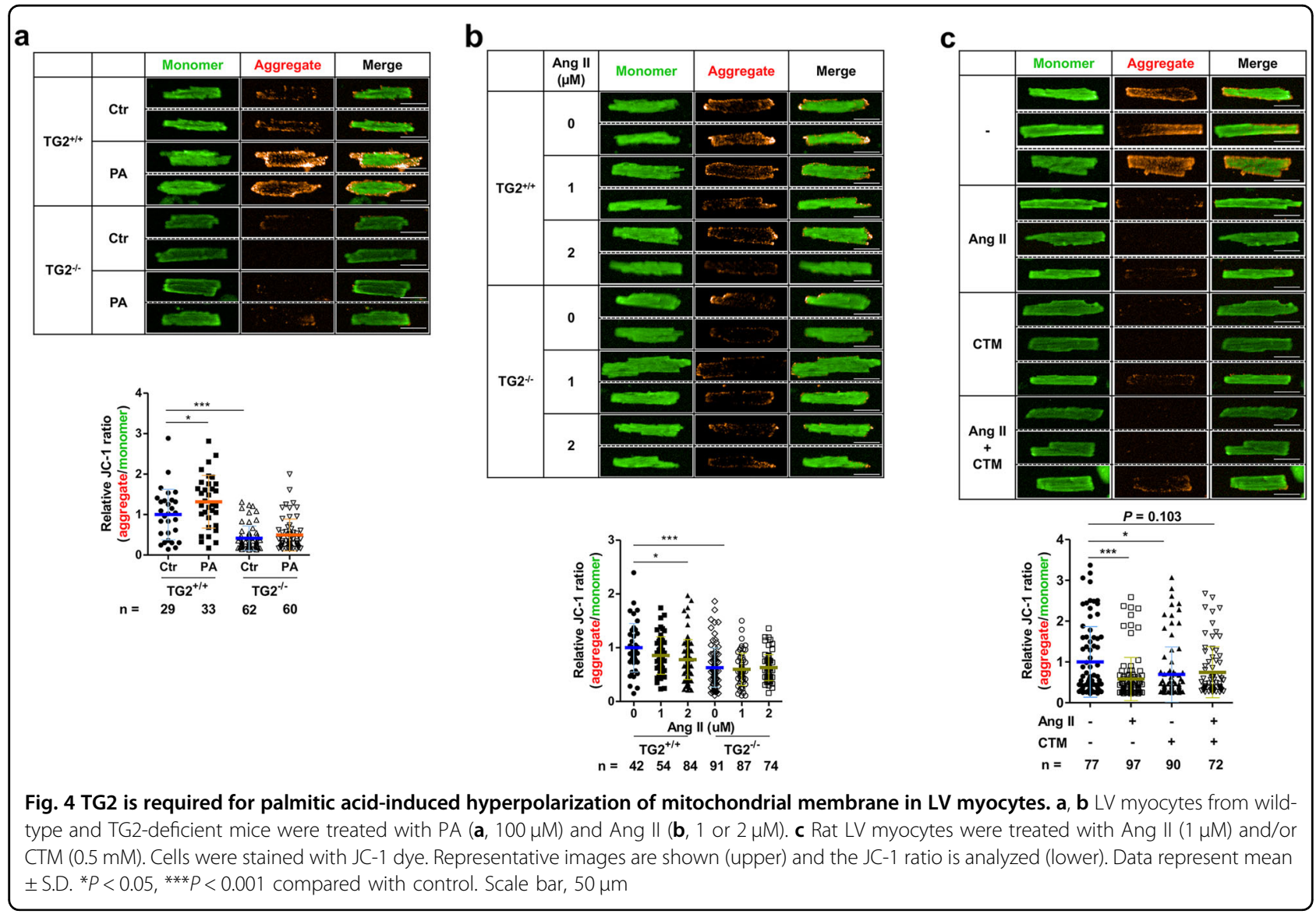

another nNOS-specific inhibitor, significantly increased TG activity in LV myocytes from shams $(P=0.004)$ and from Ang II-induced hypertensive rats $(P=0.014$, Fig. 5b). These results indicate that TG2 is inhibited by $S$-nitrosylation in Ang II-induced hypertensive myocardium.

Functionally, L-VNIO restored PA-dependent increase in LV myocyte contraction in Ang II rats $(P=0.005$, $\mathrm{L}-\mathrm{VNIO}$ vs. L-VNIO $\rightarrow \mathrm{PA}, n=3$ ). Conversely, inhibition of TG2 with cystamine abolished PA-dependent increase in myocyte contraction induced by L-VNIO in Ang II rats $(P=0.4, \quad \mathrm{~L}-\mathrm{VNIO} \quad$ vs. $\quad \mathrm{L}-\mathrm{VNIO} \rightarrow \mathrm{PA} \rightarrow$ cystamine, $n=3$, Fig. $5 \mathrm{c}, \mathrm{d})$. Moreover, cystamine pretreatment prevented PA-induced increase in myocyte contraction in the presence of $\mathrm{L}-\mathrm{VNIO}$ in Ang II rats $(P=0.7$, for $\mathrm{L}-\mathrm{VNIO} \rightarrow$ cystamine and $\mathrm{L}-\mathrm{VNIO} \rightarrow$ cystamine $\rightarrow \mathrm{PA}$, $n=3$, Fig. $5 \mathrm{c}, \mathrm{d}$ ), confirming that TG2 $S$-nitrosylation is responsible for impaired PA-dependent myocyte contraction in Ang II-treated hypertensive rats. These results indicate that $S$-nitrosylation of TG2 may represent one of the mechanisms underlying the contractile dysfunction and decreased fatty acid utilization observed in the myocardium under pressure overload.

\section{Fatty acid induces an increase of intracellular $\mathrm{Ca}^{2+}$ in LV myocytes}

Intracellular $\mathrm{Ca}^{2+}\left(\left[\mathrm{Ca}^{2+}\right]_{\mathrm{i}}\right)$ is a critical regulator of TG2 activity. To explore the mechanism for PA-induced TG2 activation, we measured $\left(\left[\mathrm{Ca}^{2+}\right]_{\mathrm{i}}\right)$ in $\mathrm{LV}$ myocytes from normal and Ang II-treated rats after PA supplementation. As shown in Fig. $6 \mathrm{a}$, the peak amplitude of $\left[\mathrm{Ca}^{2+}\right]_{\mathrm{i}}$ was significantly increased and the time constant of $\left[\mathrm{Ca}^{2+}\right]_{i}$ decay (tau) was significantly lower in Ang II rats when compared to those in shams. As a summation of these effect, total $\left[\mathrm{Ca}^{2+}\right]_{\mathrm{i}}$ (integral of $\left[\mathrm{Ca}^{2+}\right]_{\mathrm{i}}$ ) was significantly increased in Ang II rats $(P=0.02$, Fig. $6 \mathrm{~b})$. When treated with PA, peak amplitude of $\left[\mathrm{Ca}^{2+}\right]_{i}$ was not changed in both sham and Ang II rats $(P=0.5, n=33$ for shams and $P=0.5, n=27$ for Ang II rats). However, tau was increased with PA in both groups $(P<0.001, n=33$ in shams and $P=0.01, n=27$ in Ang II rats, Fig. 6a), resulting in significant increase of total $\left[\mathrm{Ca}^{2+}\right]_{\mathrm{i}}$ in shams and Ang II rats $(P=0.04$, Fig. $6 \mathrm{~b})$. These results indicate that TG2 is activated by PA-induced increase of $\left[\mathrm{Ca}^{2+}\right]_{\mathrm{i}}$ in sham myocytes, whereas TG2 is not activated by PA in Ang II rats despite of significantly higher $\left[\mathrm{Ca}^{2+}\right]_{\mathrm{i}}$, supporting the mechanism for TG2 inhibition by $S$-nitrosylation. To further confirm these results, we examine the 

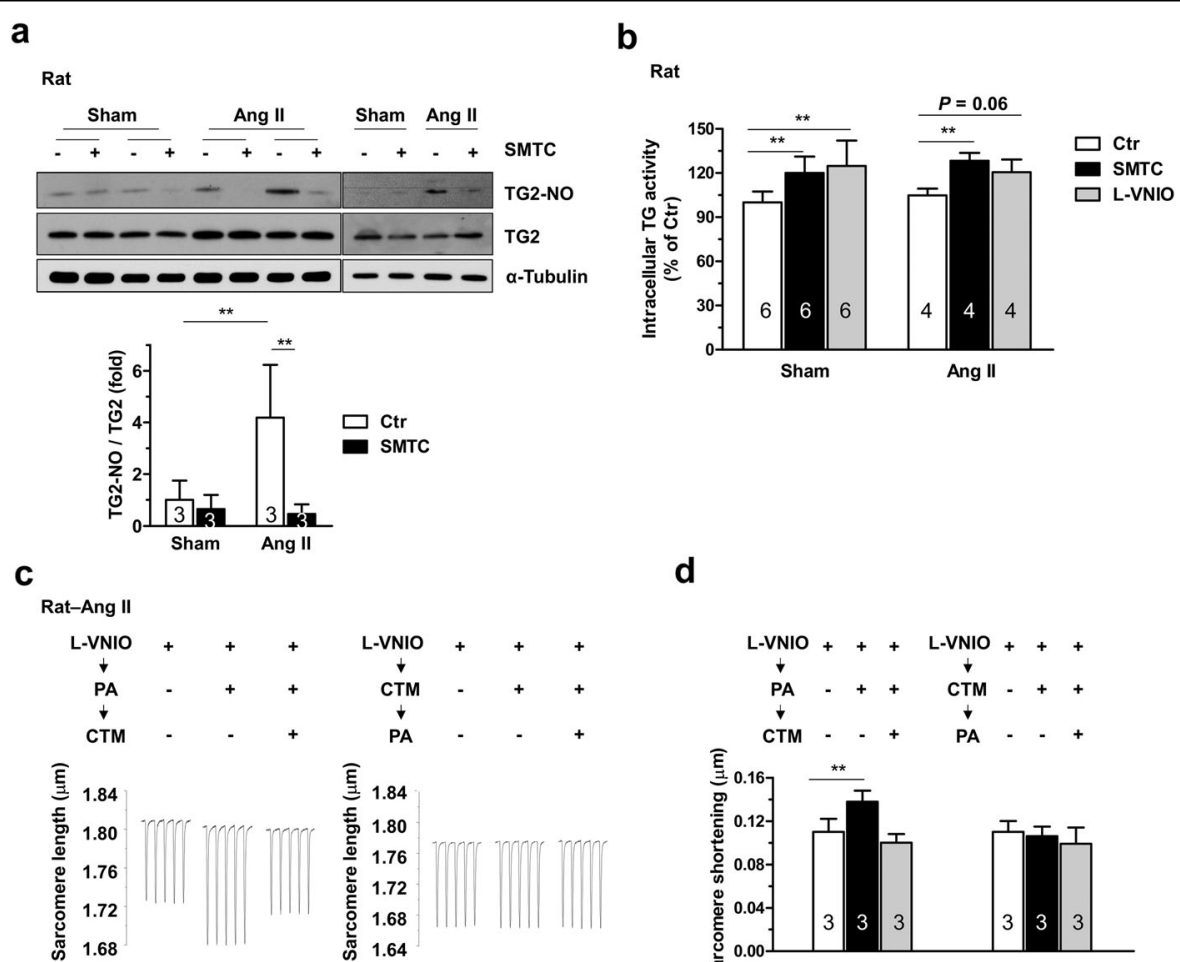

d

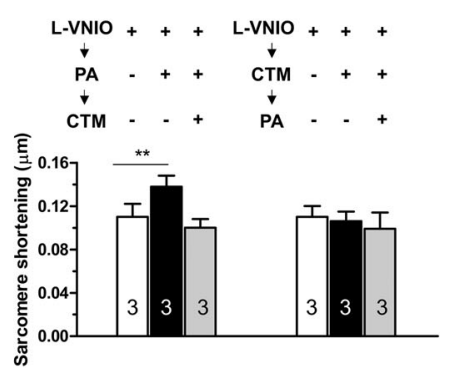

Fig. 5 S-nitrosylated TG2 is increased in cardiomyocytes from Ang II-treated rats. a Immunoblot showing S-nitrosylated TG2 (TG2-SNO) levels on treatment with SMTC (100 nM), a nNOS inhibitor, in LV myocytes from three sham and three Ang II-treated rats (left). Signals were quantified by densitometry and normalized to total TG2 levels (right, TG2-SNO/TG2). b Effects of SMTC (100 nM) or L-VNIO (100 $\mu$ M) on TG2 activity in LV myocytes from sham and Ang II-treated rats. c, d Effects of L-VNIO and/or CTM on PA-dependent contraction in LV myocytes from Ang II-treated rats. A representative graph is shown $\mathbf{c}$. The difference between diastolic and systolic sarcomere length ( $\triangle$ sarcomere shortening) was compared (d). Data represent mean \pm S.D. ${ }^{* *} P<0.01$ compared with control

effect of SMTC on total $\left[\mathrm{Ca}^{2+}\right]_{\mathrm{i}}$ in LV myocytes. Although SMTC treatment significantly increased the amplitude of $\left[\mathrm{Ca}^{2+}\right]_{\mathrm{i}}$ and decreased tau of $\left[\mathrm{Ca}^{2+}\right]_{\mathrm{i}}$ in sham myocytes $(P$ $<0.001, n=35$, Fig. $6 \mathrm{c})$, the integral of $\left[\mathrm{Ca}^{2+}\right]_{\mathrm{i}}$ was not changed in both sham and Ang II rats (Fig. 6d). These results indicate that TG2 is inhibited by $S$-nitrosylation in LV myocytes from Ang II-induced hypertensive myocardium.

\section{Discussion}

Decreased fatty acid utilization and contractile dysfunction are the characteristic features of pressure overload-induced cardiac hypertrophy ${ }^{2,3}$. In the present study, we investigated whether TG2 is involved in the modulation of myocyte contraction via the regulation of cardiac fuel metabolism. Our results showed that supplementation of media with fatty acid activates TG2, leading to an increase in ATP production and myocyte contractility. Moreover, we found that fatty acid-induced contractility was abolished in myocytes treated with TG2 inhibitors and in TG2-deficient myocytes. Furthermore, our data demonstrated that TG2 is inhibited by nNOSmediated $S$-nitrosylation in myocytes from Ang II- induced hypertensive heart, favoring impairment of fatty acid-induced myocyte contractility. Therefore, our findings suggest that TG2 plays a critical role in modulating myocyte contractility by promoting fatty acid oxidation and TG2 downregulation by $S$-nitrosylation leads to contractile dysfunction in LV myocytes following pressure-overload.

TG2 is ubiquitously expressed and widely localized in subcellular organelles ${ }^{4}$. Mitochondria from the TG2deficient heart tissue show reduced ATP production ${ }^{11}$. Conversely, mitochondria in TG2-overexpressed cells exhibit hyperpolarization and increased production of reactive oxygen species, implying increased oxidative metabolism $^{18}$. Therefore, TG2 functions as a positive regulator for mitochondrial oxidative metabolism. Our ex vivo results showed that fatty acid supplementation of media for isolated myocytes leads to the activation of TG2, which results in fatty acid oxidation. This regulation mechanism may potentially explain the increased dependence of myocytes on fatty acids for energy production under conditions of fatty acid availability.

Biomechanical stresses activate a number of signaling pathways, including $\mathrm{Ca}^{2+}$-calmodulin or redox-dependent 


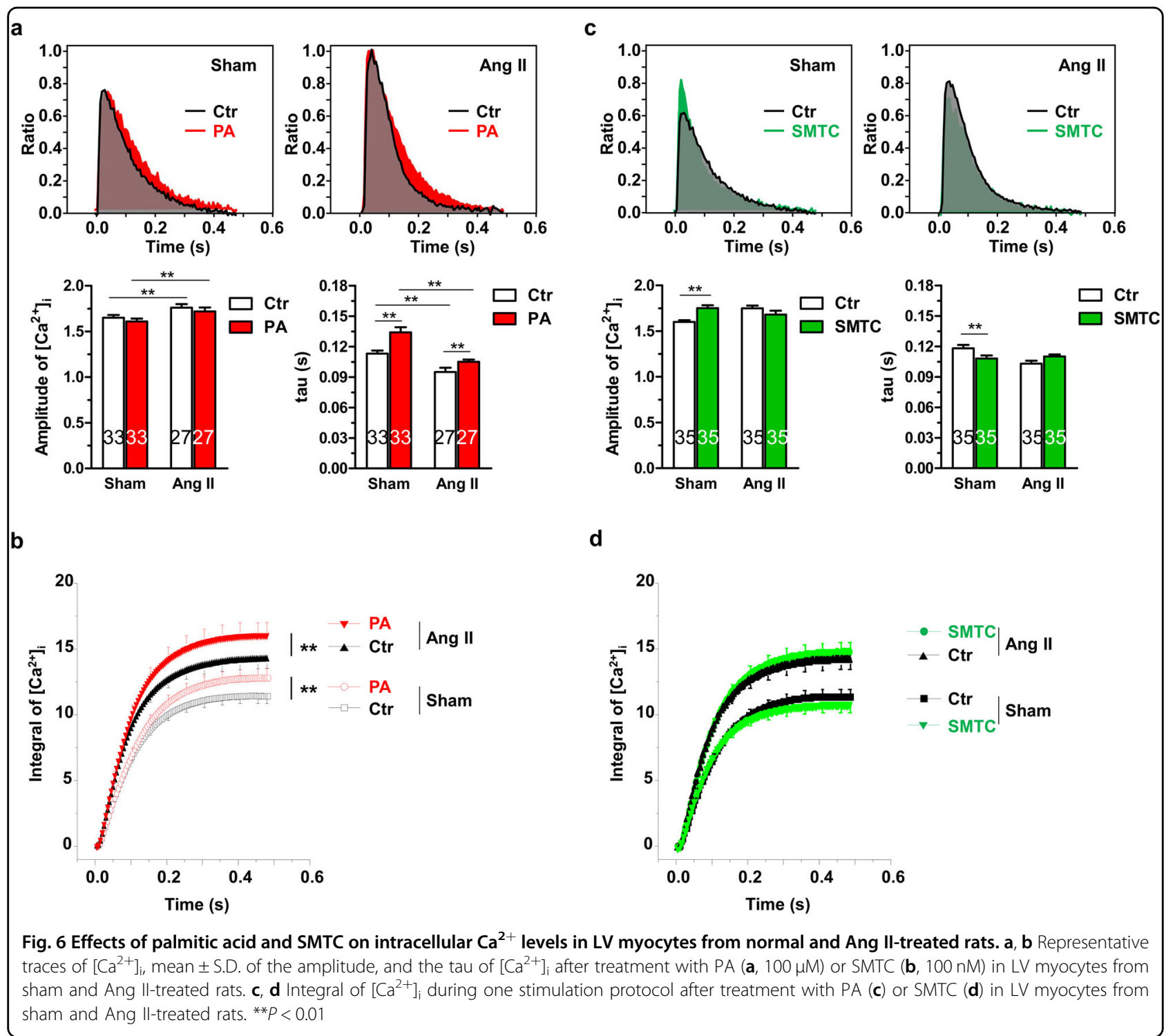

CaMKII, calcineurin, and mitogen-activated protein kinase, which activate and translocate the transcription factors, such as GATA4, nuclear factor (NF) of activated $\mathrm{T}$ cells, myocyte enhancer factor-2, histone deacetylase and signal transducer and activator of transcription factor, to the nucleus, leading to cardiac hypertrophy by increasing fetal gene expression and protein synthesis ${ }^{19}$. In particular, intracellular $\mathrm{Ca}^{2+}$, an important upstream regulator for these signaling pathway, is increased through activation of transient receptor potential channel C, Gprotein coupled receptor, sodium-calcium exchanger, and L-type calcium channel ${ }^{19}$. Thus, in myocytes under mechanical stressed conditions, increased intracellular $\mathrm{Ca}^{2}$ ${ }^{+}$level activates TG2, which in turn inhibits apoptosis by crosslinking caspase $3^{20}$, induces inflammation by $\mathrm{NFKB}$ and cyclooxygenase $2(\mathrm{COX}-2)$ activation $^{7,21}$, increases extracellular matrix by crosslinking collagen $^{22}$, and regulates fatty acid metabolism by promoting ATP production as shown in this study. Consequently, our results suggest that TG2 is a downstream effector of hypertrophic signaling in response to biomechanical stress and may play a compensatory role in the contractile dysfunction.

Isolated LV myocytes showed basal levels of TG2 activity in contrast to non-contractile cells where TG2 is inactive under normal condition ${ }^{23}$. It is likely that TG2 is activated in cardiomyocytes during the contraction, possibly by $\mathrm{Ca}^{2+}$ released from sarcoplasmic reticulum. Moreover, the processes of myocyte isolation could activate TG2 in LV myocytes since matrix deprivation by detachment induces intracellular $\mathrm{Ca}^{2+}$ increase ${ }^{24}$. However, TG2 inhibitor studies revealed that the fatty acidmediated activity of TG2 was involved in LV myocyte contractility, but its basal activity was not (Fig. 2a, b). These results imply that TG2 is differently activated in a 
context-dependent manner and fatty acid-dependent TG2 activity is only required for increasing cardiomyocyte contractility.

$S$-nitrosylation is involved in the modulation of the activities of a number of cardiac proteins, including mitochondrial complex I, hypoxia-induced factor- $1 \alpha, \alpha-$ ketoglutarate dehydrogenase, and caspase- 3 , thereby protecting myocytes from various insults such as ischemia-reperfusion injury ${ }^{13,25}$. Although $S$-nitrosylation has been known to inhibit TG2 activity ${ }^{16}$, its biological significance in cardiomyocytes has not been elucidated. Transgenic mice overexpressing TG2 in the heart exhibit ventricular hypertrophy and an increase in cardiomyocyte apoptosis and fibrosis via the upregulation of COX-2 expression ${ }^{7}$. Moreover, TG2 activity in blood vessels increases with advancing age due to decreased $S$ nitrosylation, leading to vascular stiffness ${ }^{8}$. Therefore, the regulation of TG2 activity by $S$-nitrosylation may prevent aberrant activation. In the present study, we found that TG2 is $S$-nitrosylated even in normal myocytes. The levels of $S$-nitrosylated TG2 significantly increased in LV myocytes from hypertensive rats, which show no increase in ATP production and myocyte contractility in response to fatty acid supplementation. Our findings suggest that $S$ nitrosylation is an important regulatory mechanism involved in the maintenance of optimal TG2 activity for the prevention of apoptosis and fibrosis in myocytes of the pressure-overloaded heart. In addition, our results demonstrated that nNOS is responsible for $S$-nitroylation of myocyte TG2. Treatment with nNOS inhibitors improved PA-dependent contraction in LV myocytes from Ang II-treated hypertensive rats by reducing the levels of S-nitrosylated TG2 (Fig. 5). Moreover, nNOSdeficient mice have been reported to exhibit increased cardiac performance ${ }^{26}$, suggesting that nNOS-mediated TG2 $S$-nitrosylation might play a pivotal role in the development of contractile dysfunction of heart. Thus inhibition of nNOS-mediated TG2 $S$-nitrosylation could be a novel therapeutic strategy for prevention of pressureoverloaded heart failure.

Of note, our data showed that TG2 expression levels increased in LV myocytes under the Ang II-induced hypertensive condition, compared to sham controls (Fig. 5a). Ang II has been reported to activate NFkB signaling pathway in cardiac myocytes, inducing cardiac hypertrophy and/or cardiac remodeling ${ }^{27}$, whereas TG2 is known as a target gene of $\mathrm{NFK}^{28}{ }^{28}$. Thus it is postulated that the levels of TG2 in LV myocytes from the hypertensive hearts were upregulated via the activation of $\mathrm{NFKB}$ signal, leading to increased intracellular TG activity. Given that nNOS is localized in the sarcoplasmic reticulum and mitochondria ${ }^{29,30}, S$-nitrosylation of TG2 might have occurred in these subcellular organelles, thereby inhibiting the enzymatic activity. Further studies are required for elucidating the precise subcellular location where TG2 is $S$-nitrosylated and for identifying TG2 substrate proteins in myocytes from the hypertensive hearts. Unexpectedly, basal levels of sarcomere shortening of $\mathrm{TG}^{-1-}$ myocytes were higher than those of wild-type myocytes (Fig. 2c). It is plausible that TG2 may have another role in the regulation of myocyte contractility, in addition to potentiation of PA-stimulated sarcomere shortening. Other possibility is compensatory phenotype of $\mathrm{TG}^{-1-}$ myocytes. To explain this phenotype, further studies will be needed such as comparison of the transcriptomes of wild-type and TG2 $2^{-1-}$ myocytes.

In summary, our results indicate a novel role for TG2 in the regulation of cardiac contraction, which is impaired by $S$-nitrosylation in myocytes under pressure overload, thereby identifying this enzyme as an important player in the development of contractile dysfunction in the heart under mechanical stress.

\section{Acknowledgements}

The present study was funded by the National Research Foundation (NRF) of Korea (Basic Science Research Program (2010-0022-819, NRF2013R1A1A2065273, NRF-2014R1A2A2A01006731, NRF-

2017R1D1A1B03035059, and NRF-2017M3A9B4061890)), the Korea Healthcare Technology R\&D Project of Ministry of Health and Welfare of Korea

(HI14C3339), the Brain Korea 21 (BK21) PLUS program through the NRF funded by the Ministry of Education of Korea, the Korean Society of Hypertension, the SK Telecom Research Fund (3420130290) and National Natural Science Foundation of China (NSFC, 31460265, and NSFC 31660284).

\section{Author details}

'Department of Biochemistry and Molecular Biology, Seoul National University College of Medicine, Jongno-gu, Seoul, Republic of Korea. Institute of HumanEnvironment Interface Biology, Seoul National University College of Medicine, Jongno-gu, Seoul, Republic of Korea. ${ }^{3}$ Department of Physiology, Ischemic/ Hypoxic Disease Institute, Seoul National University College of Medicine, Jongno-gu, Seoul, Republic of Korea. ${ }^{4}$ Yan Bian University Hospital, Yan Ji, Ji Lin Province, China. Institute of Cardiovascular Sciences, University of Manchester, Manchester, UK

Conflict of interest

The authors declare that they have no conflict of interest.

\section{Publisher's note}

Springer Nature remains neutral with regard to jurisdictional claims in published maps and institutional affiliations.

Received: 10 January 2017 Revised: 30 October 2017 Accepted: 13 November 2017.

Published online: 6 April 2018

\footnotetext{
References

1. Neely, J. R. \& Morgan, H. E. Relationship between carbohydrate and lipid metabolism and the energy balance of heart muscle. Annu. Rev. Physiol. 36, 413-459 (1974).

2. Kolwicz, S. C. Jr., Purohit, S. \& Tian, R. Cardiac metabolism and its interactions with contraction, growth, and survival of cardiomyocytes. Circ. Res. 113, 603-616 (2013).

3. Chatham, J. C. \& Young, M. E. Metabolic remodeling in the hypertrophic heart: fuel for thought. Circ. Res. 111, 666-668 (2012).
} 
4. Lorand, L. \& Graham, R. M. Transglutaminases: crosslinking enzymes with pleiotropic functions. Nat. Rev. Mol. Cell Biol. 4, 140-156 (2003).

5. Facchiano, F., Facchiano, A. \& Facchiano, A. M. The role of transglutaminase-2 and its substrates in human diseases. Front. Biosci. 11, 1758-1773 (2006).

6. lismaa, S. E., Mearns, B. M., Lorand, L. \& Graham, R. M. Transglutaminases and disease: lessons from genetically engineered mouse models and inherited disorders. Physiol. Rev. 89, 991-1023 (2009).

7. Zhang, Z. et al. COX-2-dependent cardiac failure in Gh/tTG transgenic mice. Circ. Res. 92, 1153-1161 (2003).

8. Santhanam, L. et al. Decreased S-nitrosylation of tissue transglutaminase contributes to age-related increases in vascular stiffness. Circ. Res. 107, 117-125 (2010).

9. Sane, D. C., Kontos, J. L. \& Greenberg, C. S. Roles of transglutaminases in cardiac and vascular diseases. Front. Biosci. 12, 2530-2545 (2007).

10. Min, S. K. et al. Intimal hyperplasia in loop-injured carotid arteries is attenuated in transglutaminase 2-null mice. J. Korean Med. Sci. 29, 363-369 (2014).

11. Szondy, Z. et al. Tissue transglutaminase (TG2) protects cardiomyocytes against ischemia/reperfusion injury by regulating ATP synthesis. Cell Death Differ. 13, 1827-1829 (2006).

12. Malorni, W. et al. The adenine nucleotide translocator 1 acts as a type 2 transglutaminase substrate: implications for mitochondrial-dependent apoptosis. Cell Death Differ. 16, 1480-1492 (2009).

13. Sun, J. \& Murphy, E. Protein S-nitrosylation and cardioprotection. Circ. Res. 106, 285-296 (2010)

14. Jin, C. Z. et al. Myofilament $\mathrm{Ca} 2+$ desensitization mediates positive lusitropic effect of neuronal nitric oxide synthase in left ventricular myocytes from murine hypertensive heart. J. Mol. Cell. Cardiol. 60, 107-115 (2013).

15. Damy, T. et al. Up-regulation of cardiac nitric oxide synthase 1-derived nitric oxide after myocardial infarction in senescent rats. FASEB J. 17, 1934-1936 (2003).

16. Lai, T. S. et al. Calcium regulates S-nitrosylation, denitrosylation, and activity of tissue transglutaminase. Biochemistry 40, 4904-4910 (2001).

17. De Laurenzi, V. \& Melino, G. Gene disruption of tissue transglutaminase. Mol. Cell. Biol. 21, 148-155 (2001).
18. Piacentini, M. et al. Transglutaminase overexpression sensitizes neuronal cell lines to apoptosis by increasing mitochondrial membrane potential and cellular oxidative stress. J. Neurochem. 81, 1061-1072 (2002).

19. Balakumar, P. \& Jagadeesh, G. Multifarious molecular signaling cascades of cardiac hypertrophy: can the muddy waters be cleared? Pharmacol. Res. 62 , 365-383 (2010).

20. Yamaguchi, H. \& Wang, H. G. Tissue transglutaminase serves as an inhibitor of apoptosis by cross-linking caspase 3 in thapsigargin-treated cells. Mol. Cell. Biol. 26, 569-579 (2006)

21. Falasca, L. et al. Transglutaminase type $\|$ is involved in the pathogenesis of endotoxic shock. J. Immunol. 180, 2616-2624 (2008).

22. Collighan, R. J. \& Griffin, M. Transglutaminase 2 cross-linking of matrix proteins: biological significance and medical applications. Amino Acids 36, 659-670 (2009).

23. Shin, D. M. et al. Cell type-specific activation of intracellular transglutaminase 2 by oxidative stress or ultraviolet irradiation: implications of transglutaminase 2 in age-related cataractogenesis. J. Biol. Chem. 279, 15032-15039 (2004).

24. Sundararaman, A., Amirtham, U. \& Rangarajan, A. Calcium-oxidant signaling network regulates AMP-activated protein kinase (AMPK) activation upon matrix deprivation. J. Biol. Chem. 291, 14410-14429 (2016).

25. Hess, D. T. \& Stamler, J. S. Regulation by S-nitrosylation of protein posttranslational modification. J. Biol. Chem. 287, 4411-4418 (2012).

26. Dawson, D. et al. nNOS gene deletion exacerbates pathological left ventricular remodeling and functional deterioration after myocardial infarction. Circulation 112, 3729-3737 (2005).

27. Rouet-Benzineb, P., Gontero, B., Dreyfus, P. \& Lafuma, C. Angiotensin II induces nuclear factor- kappa B activation in cultured neonatal rat cardiomyocytes through protein kinase C signaling pathway. J. Mol. Cell. Cardiol. 32, 1767-1778 (2000).

28. Kuncio, G. S. et al. TNF-alpha modulates expression of the tissue transglutaminase gene in liver cells. Am. J. Physiol. 274, G240-G245 (1998).

29. Xu, K. Y., Huso, D. L., Dawson, T. M., Bredt, D. S. \& Becker, L. C. Nitric oxide synthase in cardiac sarcoplasmic reticulum. Proc. Natl. Acad. Sci. USA 96, 657-662 (1999).

30. Kanai, A. J. et al. Identification of a neuronal nitric oxide synthase in isolated cardiac mitochondria using electrochemical detection. Proc. Natl. Acad. Sci. USA 98, 14126-14131 (2001). 